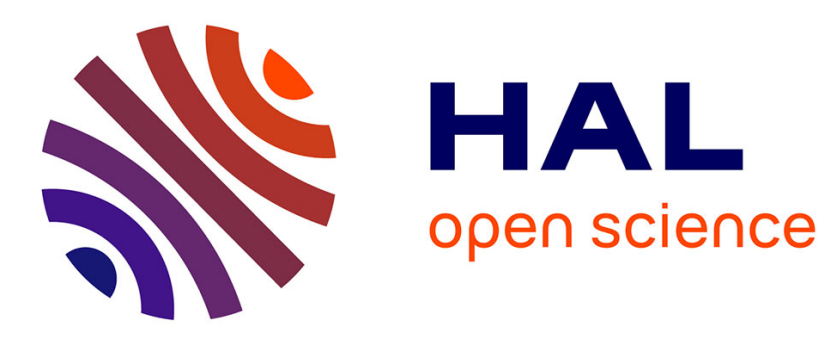

\title{
The elephant mode between two rotating disks
}

\author{
B. Viaud, E. Serre, Jean-Marc Chomaz
}

\section{To cite this version:}

B. Viaud, E. Serre, Jean-Marc Chomaz. The elephant mode between two rotating disks. Journal of Fluid Mechanics, 2008, 598 (mars), pp.451-464. 10.1017/s0022112007009962 . hal-01022811

\section{HAL Id: hal-01022811 \\ https://hal-polytechnique.archives-ouvertes.fr/hal-01022811}

Submitted on $20 \mathrm{Jul} 2014$

HAL is a multi-disciplinary open access archive for the deposit and dissemination of scientific research documents, whether they are published or not. The documents may come from teaching and research institutions in France or abroad, or from public or private research centers.
L'archive ouverte pluridisciplinaire HAL, est destinée au dépôt et à la diffusion de documents scientifiques de niveau recherche, publiés ou non, émanant des établissements d'enseignement et de recherche français ou étrangers, des laboratoires publics ou privés. 


\title{
The elephant mode between two rotating disks
}

\author{
BERTRAND VIAUD ${ }^{1}$, ERIC SERRE ${ }^{2}$ AND \\ JEAN-MAR C CHOMAZ 3 \\ ${ }^{1}$ Centre de Recherche de l'Armée de l'Air, CReA BA701 13661 Salon de Provence, France \\ ${ }^{2}$ Laboratoire MSNM-GP, CNRS Universités Aix Marseille, IMT Chateau-Gombert \\ 13451 Marseille, France \\ ${ }^{3}$ Laboratoire d'Hydrodynamique-LadHyX, CNRS-Ecole Polytechnique, F-91128 Palaiseau, France
}

(Received 21 May 2007 and in revised form 26 November 2007)

Spectral direct numerical simulations (DNS) are carried out for a source-sink flow in an annular cavity between two co-rotating disks. When the Reynolds number based on the forced inflow is increased, a self-sustained crossflow instability of finite amplitude is observed. We show that this nonlinear global mode is made up of a front located at the upstream boundary of the absolutely unstable domain, followed by a saturated spiral mode, and that its properties are in good agreement with results of the local stability theory. This structure is characteristic of the so-called elephant mode of Pier \& Huerre (J. Fluid Mech. vol. 435, 2001, p. 145). The global bifurcation is subcritical since only large-amplitude initial perturbations are found to trigger the elephant mode. Small-amplitude perturbations induce a long-lasting transient growth but lead eventually to a damped linear global mode, showing that non-parallel effects counteract the absolute instability and restabilize the flow. A similar linear global stabilization due to non-parallel effects has been found in the case of the flow above a single rotating disk. For the single-disk geometry, the existence of an elephant mode would imply, together with results of Davies \& Carpenter (2003) a subcritical global instability, which has not yet been demonstrated. Although the present geometry differs from the single-disk case, the existence of a subcritical global bifurcation is now established, allowing a precise analysis of the transition scenarios.

\section{Introduction}

The flow between two co-rotating disks, entering at the hub and exiting at the rim, provides a simple model of technological devices such as turbomachinery (see Owen \& Rogers 1995). As discussed by Hide (1968), this configuration is also relevant to geophysical flows, since for large rotation rate it is composed of two Ekman boundary layers separated by a core in quasi-solid-body rotation. The viscous flow near a single rotating wall has long served as a prototype flow for three-dimensional boundary layers, as it exhibits an inflectional velocity profile and an inviscid crossflow instability. Since the discovery of the exact similarity solution of von Kármán (von Kármán 1921), many works have been devoted to the analysis of the transition process to turbulence in these thin boundary layers, see the review in Crespo del Arco et al. (2005). Linear stability analysis has revealed two generic forms of instability, referred as type I (crossflow) and type II (viscous). A type III found in the spatial theory by Mack (1985) seems to transport energy inward, and consequently is heavily damped. It was later found to correspond to the upstream branch of the type I convective mode. 
Interest in this flow was renewed after Lingwood $(1995,1996,1997)$ found that the crossflow instability undergoes a transition from convective to absolute behaviour at a Reynolds number not far below its value characterizing the onset of turbulence in the experiments. Lingwood suggested that type I and type III were the coalescing branches at the origin of the type I absolute mode. Numerical investigations of the linear stability equations conducted by Turkyilmazoglu \& Gajjar (2000) and of the linearized Navier-Stokes equations by Davies \& Carpenter (2003) yielded results consistent with those of Lingwood when the base flow is parallel, confirming the existence of an absolute instability. However, Turkyilmazoglu \& Gajjar (2000) argued that if the nonparallel effects were taken into account, the transition from a convective to an absolute regime might occur at a significantly higher Reynolds number, possibly above the experimental onset of turbulence. Moreover, they investigated the coalescence of type I and II modes (propagating in the same direction), and found that they are at the origin of a direct spatial resonance at a Reynolds number (445) significantly lower than the one associated with the absolute instability threshold (507). This resonance results in a short-term algebraic growth that could promote nonlinearity and transition, before the occurrence of absolute instability. As the resonance Reynolds number predicted with the parallel-flow assumption (445) is sufficiently lower than the Reynolds number at the onset of turbulence (513), this direct spatial resonance scenario was suggested to remain valid even though non-parallel effects might lead to a downstream shift of the resonance location.

Davies \& Carpenter (2003) studied the linear impulse response for different azimuthal modes and investigated the validity of the parallel-flow assumption. When considering the non-parallel flow, they obtained results consistent with the experimental observations of Lingwood. Whereas transient absolute behaviour was observed it was not sustained in time and eventually convective behaviour dominated as the perturbations locally tended to relax toward zero. They thus concluded that there was no evidence of the absolute instability giving rise to a global oscillator. Davies, Thomas \& Carpenter (2007) showed later that detuning arising from the radial variations of the absolute frequency, a consequence of the non-parallel effects, might be sufficiently stabilizing to maintain linear global stability, even in the presence of absolute instability.

But as proposed in the context of the inhomogeneous envelope equation by Couairon \& Chomaz (1997, 1999a, b), Meunier et al. (1997), Tobias, Proctor \& Knobloch (1997, 1998), Pier et al. (1998), Bassom, Kuzanyan \& Soward (1999), when nonlinearities are taken into account, the presence of an absolutely unstable region is a sufficient condition for the existence of a fully nonlinear global mode. In the particular case of weakly non-parallel flows presenting a finite region of absolute instability embedded in a convectively unstable domain, the nonlinear global mode takes the form of a steep front located at the upstream transition point from convective to absolute instability, separating evanescent perturbations upstream from a saturated nonlinear wave downstream: the elephant mode described by Pier et al. (1998) and Bassom et al. (1999) and named later in Pier \& Huerre (2001b). As discussed in Couairon \& Chomaz (2001) the elephant mode may be pushed or pulled, depending whether the front velocity is nonlinearly or linearly selected (van Saarloos 2003). The front velocity in parallel flow may be nonlinearly selected and the front is then said to be pushed. This can happen when the local bifurcation is subcritical, i.e. when the nonlinearities locally destabilize the flow. If so, when non-parallel effects are taken into account, a pushed elephant mode may exist even when the flow is everywhere locally linearly stable or convectively unstable. Such flows are globally linearly stable, and 
bifurcations to the pulled elephant mode are intrinsically subcritical. In the majority of cases studied so far, the front velocity is linearly selected and the existence of the elephant mode is linked to the presence of an absolutely unstable region in the flow, the upstream part of the absolute domain then pulling the elephant mode.

However, and due to non-parallel effects, this absolutely unstable region may not be sufficient to create a linear global mode. This body of work opens the possibility for a flow to remain linearly globally stable, while supporting a fully nonlinear global mode, i.e. to be globally subcritical even if the local instability is supercritical (Chomaz \& Couairon 1999). In that spirit Pier (2003) proposed that an elephant mode develops over a single rotating disk, and studied the local secondary stability of the saturated type I wave that would then be present downstream of the front when the primary instability is absolute. Indeed, as initially proposed by Huerre (1988) as a generic shortcut on the route to turbulence (and developed by Brancher \& Chomaz 1997; Couairon \& Chomaz 1999b; Chomaz, Couairon \& Julien 1999; Koch 2002), Pier (2003) showed that the saturated type I absolute mode is subject to secondary absolute instability at a Reynolds number lower than the one for which the primary instability is absolute. Pier concludes that the naturally selected structure is thus dynamically unstable and gives way to a turbulent regime. In the single-disk case however, the existence of the nonlinear global mode proposed by Pier (2003) has not yet been confirmed.

The present paper tackles a different but related geometry, and investigates through direct numerical simulation (DNS) the local and global linear stability properties of the flow, as well as its fully nonlinear and non-parallel dynamics. A flow is forced between two parallel disks rotating around the same axis and at the same velocity. In this configuration, as the mass flow rate and the rotation speed can be chosen separately, Rossby and Reynolds numbers are controlled independently. When the gap between the two disks is large with respect to the boundary-layer scale $\delta, \delta / h=0.035$, the flow at any radial station is made up of a core locally in solid-body rotation sandwiched between two thin Ekman layers, and is locally equivalent at leading order to that found above a single disk in a rotating tank.

The configuration is detailed in $\S 2$, together with the mathematical and numerical modelling. Section 3.1 presents the axisymmetric base flow and investigates its local stability properties. They are shown to match those obtained by Lingwood (1997) for the single-disk case. Global stability is the subject of $\S 3.2$ and $\S 3.3$. The existence of a nonlinear global mode, matching the elephant mode theory, is shown in $\S 3.2$. Then, $\S 3.3$ establishes the subcriticality of this global bifurcation. The relevance of this configuration to the single-disk case is further discussed in $\S 4$.

\section{Modelling and numerical method}

\subsection{Rotating-disk flow and non-dimensional equations}

The geometrical model (figure 1) corresponds to two parallel disks distance $h^{*}$ apart enclosing an annular domain of radial extent $\Delta R^{*}=R_{\text {out }}^{*}-R_{\text {in }}^{*}$, with the star denoting dimensional variables. The two disks rotate at uniform angular velocity $\boldsymbol{\Omega}=\Omega \boldsymbol{e}_{z}$, $\boldsymbol{e}_{z}$ being the unit vector in the axial direction. The geometrical parameters are the curvature $R_{m}=\left(R_{\text {in }}^{*}+R_{\text {out }}^{*}\right) / \Delta R^{*}$, and the aspect ratio $L=\Delta R^{*} /\left(h^{*}\right)$.

The incompressible fluid motion is governed by the three-dimensional NavierStokes equations, written in the rotating frame associated with the disks. The equations are made non-dimensional using $h^{*}, \Omega^{-1}, \Omega h^{*}$ as characteristic scales for length, time and velocity, respectively. A flow is imposed at the inlet, with a mass flow rate $Q^{*}$, 


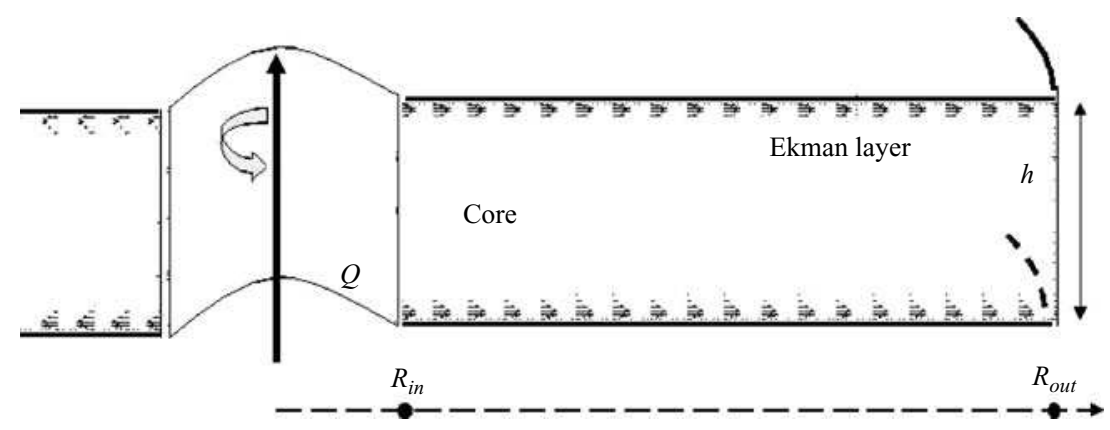

FIGURE 1. Sketch of the annular cavity. Velocity vector field in the $(r, z)$-plane depicts the radial velocity of the base flow.

which once made non-dimensional becomes the control parameter $C_{w}=Q^{*} /\left(\nu R_{\text {out }}^{*}\right)$, $v$ being the kinematic viscosity.

For the flow above a single disk, in the asymptotic limit of high rotation rates $\Omega$, the Navier-Stokes equations are linear at leading order, the Coriolis force dominating over the nonlinear advection terms. They then admit the exact Ekman solution, linearly proportional to the azimuthal velocity away from the disk $V_{\infty}^{*}=\Omega_{f} r^{*}$ which is an increasing function of $r^{*}$.

$$
u^{*}=V_{\infty}^{*}\left(r^{*}\right) \exp \left(-z^{*} / \delta^{*}\right) \sin \frac{z^{*}}{\delta^{*}}, \quad v^{*}=V_{\infty}^{*}\left(r^{*}\right)\left(1-\exp \left(-z^{*} / \delta^{*}\right) \cos \frac{z^{*}}{\delta^{*}}\right),
$$

where $\delta^{*}=\sqrt{\nu / \Omega}$ is the characteristic length scale of the Ekman boundary layer, and $u^{*}$ and $v^{*}$ are the radial and azimuthal components of the velocity, respectively. The axial component $w^{*}$, proportional to $\left(\delta^{*} / r^{*}\right) V_{\infty}^{*}$, is asymptotically smaller than $u^{*}$ and $v^{*}$ when $\left(\delta^{*} / r^{*}\right)$ goes to zero, and is usually taken to be zero.

The Ekman solution can be extended to the two-disk case, Serre et al. (2001), and has been shown to describe accurately the base flow when $C_{w}$ is small. It is hereafter referred to as the asymptotic solution. In this configuration $V_{\infty}^{*}$ is replaced by $V_{g}^{*}$, the azimuthal component of velocity at midheight, hereafter called geostrophic velocity. A global Reynolds number can be extracted from the ratio of the Ekman length scale and the cavity height, $R e_{h}=\left(h^{*} / \delta^{*}\right)^{2}$. For the asymptotic solution, the geostrophic velocity, negative in the rotating frame, can be determined using mass flow rate conservation, and expressed in terms of $R e_{h}$ and $C_{w}$, following $V_{g}=V_{g}^{*} /\left(\Omega h^{*}\right)=$ $-C_{w} R_{\text {out }}^{*} /\left(2 \pi r^{*} \sqrt{R e_{h}}\right)$. So the base flow is not strictly parallel, and unlike the single infinite-disk case the geostrophic velocity decreases with the radius like $1 / r$. The local Rossby number $R o=\Delta \Omega / \Omega$ is defined using $\Delta \Omega=\Omega_{f}-\Omega$, with $\Omega_{f}=V_{g}^{*} / r^{*}$ the fluid rotation rate in the core and $\widetilde{\Omega}$ the reference rotation rate of the system proposed by Lingwood (1997), $\widetilde{\Omega}=\left(\Omega_{f}+\Omega\right) / 4+\sqrt{\left(\left(\Omega_{f}+\Omega\right) / 4\right)^{2}+(\Delta \Omega)^{2} / 2}$. It compares the (nonlinear) inertial effects to the (linear) Coriolis force, so that when $R o$ goes to zero, the actual solution is close to the asymptotic one. The local Reynolds and Rossby numbers for the asymptotic solution are decreasing functions of the radial station $r$ :

$$
R e_{\delta}=\frac{-V_{g}^{*} \delta^{*}}{v}=\frac{R_{o u t} C_{w}}{2 \pi r}, \quad R o=-\frac{R e_{\delta}}{r \sqrt{R e_{h}}} .
$$

We can summarize this description of our configuration by stating that the geometry is defined by the two parameters $L$ and $R_{m}$, and the flow by $C_{w}$ and $\Omega$, the global control parameters. Alternatively it may be more convenient to replace the control 


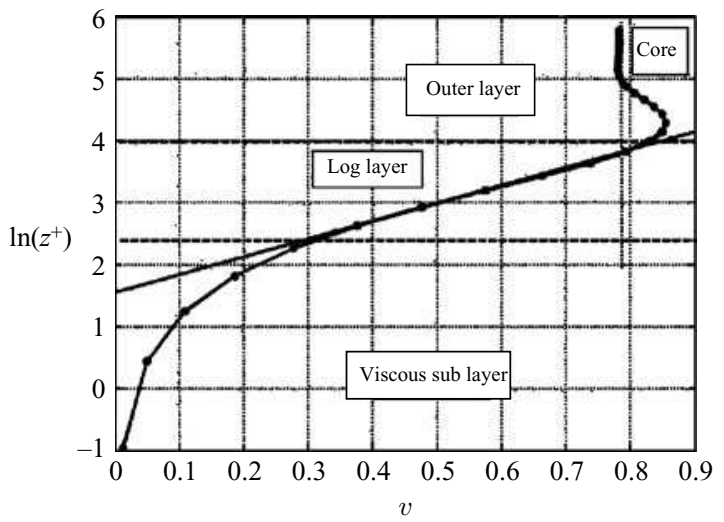

FiguRE 2. Boundary-layer structure and mesh in the axial direction. The magnitude of the velocity in the plane of the walls is plotted with respect to the natural logarithm of the wall coordinate. The circles show the mesh points.

parameter $\Omega$ by $R e_{h}$ which directly indicates the ratio of the boundary-layer thickness to the gap. Local stability is governed by $R o$ and $R e_{\delta}$, the local control parameters, which are functions of the global ones and the radial position. For a given station, stability properties depend on the magnitude of $R e_{\delta}$ with respect to its critical value, itself function of the local Rossby number $\mathrm{Ro}$.

\subsection{Numerical method}

The numerical method is based on a pseudo-spectral Tchebyshev-Fourier discretization. In the axial and radial directions Tchebyshev polynomials are used on the Gauss-Lobatto collocation points, and Fourier expansion is applied in the azimuthal direction.The density of the Gauss-Lobatto collocation points is higher near the boundaries, allowing a precise description of the boundary-layer structures, including the viscous sublayer, as shown in figure 2.

Any of the dimensionless flow variables $\Psi \in\{u, v, w, p\}$ can be expanded in the form of a truncated series, the spectral coefficients being the new unknowns. Spatial derivatives are obtained in the spectral space through multiplication by suitable matrices, whereas the advection terms are evaluated at the collocation points through fast transform between spectral and physical spaces. This pseudo-spectral discretization ensures exponential convergence of the solution, see Raspo et al. (2002).

The time scheme is semi-implicit and second-order accurate. It is a combination of an explicit treatment (second-order Adams-Bashforth) of the Coriolis and nonlinear terms, and an implicit (backward Euler) discretization for the viscous diffusive terms. The incompressibility condition is imposed through a projection algorithm. A time step involves successive resolution of three Helmholtz equations for the pressure predictor, the velocity and the pressure corrector respectively. A direct solver for these equations is used, based on a complete matrix diagonalization technique (Raspo et al. 2002).

For a cavity defined by $L=5$ and $R_{m}=9$, the number of points was progressively increased in every direction, up to mesh independence of the observed dynamics. The final mesh contained $241 \times 65 \times 320$ points in the radial, axial and azimuthal directions respectively. The associated time step was $\delta t=10^{-5}$. 


\subsection{Boundary conditions}

The definition and tuning of inflow and outflow boundary conditions was a key issue. For any numerical simulation of an open flow where perturbations may enter and leave the computational domain, inlet and outlet conditions may introduce artificial dynamics due to unphysical reflections of instability waves or spurious coupling through the pressure term. At the outlet, convective boundary conditions (Sommerfeld type) were used in order to avoid reflections of the wave packets. Such conditions have been reviewed by several authors, see Ruith, Chen \& Meiburg (2004), and it appears that a local explicit discretization with a constant advection velocity is best suited here. The outlet convection velocity, $C$, must be as close as possible to the actual local phase velocity of the wave packets, but it must also ensure mass flow rate conservation, and respect the local CFL condition. So at each new time step $n+1$ the three components imposed at the outflow are replaced according to the equation $u_{j}^{n+1}=u_{j}^{n}-C(\delta t / \delta r)\left(u_{j}^{n}-u_{j-1}^{n}\right)$. At the inlet, the three components of the velocity were prescribed and precomputed profiles were used, as no analytical solution for this configuration is known. The profile was picked up from the computation of the axisymmetric flow in a longer cavity overlapping the present one. This procedure allows us to impose a balanced flow at the inlet of the present three-dimensional highresolution computation, and therefore avoid unnecessary CPU time consumption in a region where, when the inlet condition is not balanced, fast adjustment occurs and may generate strong specific instabilities. If the usual Poiseuille profile is imposed at the inlet, it produces an entry zone preceding the Ekman layer, as shown by Hide (1968), which may be unstable, see in Crespo del Arco et al. (1996), and contaminates the rest of the flow. Similarly, imposing an inlet profile made up of two matched Ekman solutions, Serre et al. (2001), produces too much perturbation at the inlet due to the mismatch between a forced entry profile obtained from linearized equations and nonlinearity of the subsequent base flow.

At the disks the no-slip boundary condition was applied.

\section{Results}

For high enough $R e_{h}$, the axisymmetric base flow is composed of two boundary layers over the disks, separated by a central core where only the azimuthal component, corresponding to the geostrophic velocity, $V_{g}$, is non-zero (figure 3 ). As predicted by the Proudman theorem, the axial gradient of azimuthal velocity is zero in the core. The global Reynolds number was kept constant at $R e_{h}=780$, and the control parameter of the throughflow $\left(C_{w}\right)$ varied in the range $[200 ; 2500]$, inducing variations of the local parameters $R e_{\delta}$ and $R o$ according to (2.2). The local Rossby number can range from zero (Ekman solution) to about minus one (von Kármán solution), with increasing values of $C_{w}$. Moreover, for a fixed value of $C_{w}, R o$ decreases continuously like $1 / r$ over the cavity. By comparison, in the theoretical work of Lingwood, stability analysis was performed for several discrete values of the Rossby number, namely $-1,-0.8$, $-0.6,-0.4,-0.2$ and 0 .

Large local Reynolds numbers $R e_{\delta}$ are achieved through large throughflow $C_{w}$, and thus involve finite local Rossby number, so that the base flow departs from the asymptotic solution. This finite-Rossby-number effect is illustrated on figure 3 which compares the asymptotic and numerical azimuthal velocity profiles for two values of $C_{w}$, corresponding respectively at midcavity to $R o=-0.17$ and $R o=-0.8$. Lingwood (1997) took this finite-Rossby-number effect into account, by using for her linear stability analysis a base state derived from a numerical solution of the von 

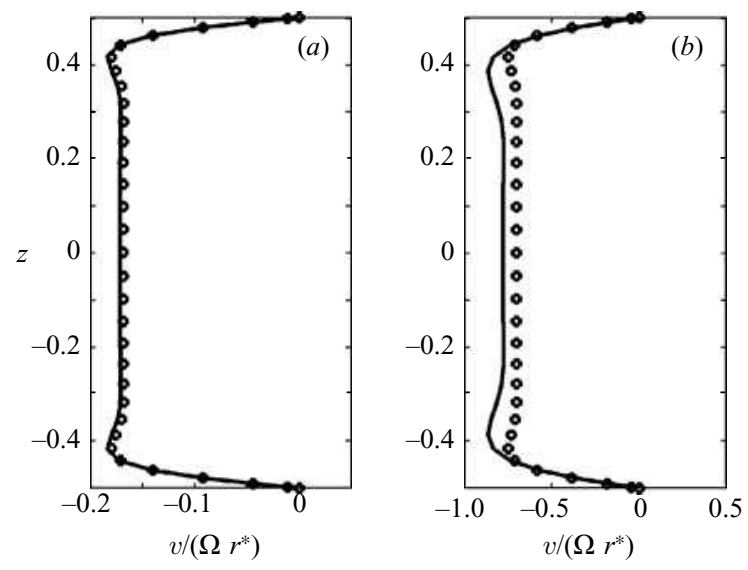

FIGURE 3. Axial profiles of the azimuthal component of the velocity at $r=22.5$, scaled by the local disk velocity $\Omega r$. (a) $R o=-0.17$ and $(b) R o=-0.80$. The solid line represents the nonlinear DNS results, and the $\bigcirc$ shows the asymptotic solution, made up of two Ekman layers.

Kármán similarity equation, extended to $R o \neq-1$. For a review of nonlinearity and non-similarity in Ekman layers see Andersen, Lautrup \& Bohr (2003).

For axisymmetric computations the solution is stable throughout the range of control parameter $C_{w} \in[200,2500]$. This result is consistent with the local stability properties of axisymmetric perturbations that are known (Lingwood 1997) to be convective. Machine-precision round-off noise might generate amplified perturbations but they are washed away. Still, this observation made when running axisymmetric computations for extremely long time, compared to the transit time in the cavity, demonstrates that the present boundary conditions, in particular at the outlet, do not introduce artificial feedback of perturbations that would lead to spurious destabilization. The converged steady axisymmetric solution was then taken as base flow for the following study.

\subsection{Local linear stability of the axisymmetric base flow}

We used the DNS code to conduct a local stability analysis of this base flow, following the method proposed by Delbende, Chomaz \& Huerre (1998). In this, velocity profiles corresponding to any chosen radial station can be extracted from the axisymmetric steady DNS solution, and extended to the whole radial extent of the computational domain, creating a strictly parallel base flow. This parallel base flow is maintained by enforcing the zero Fourier coefficient to remain constant, and the dynamics of any non-zero azimuthal wavenumber is forced by a perturbation initially localized along the radial direction. This impulse response is recorded for velocity profiles corresponding to each radial station, allowing us to extract the location $r_{C A}$ of transition from convective to absolute instability, together with the absolute frequency $\omega_{0}$ and spatial growth rate $k_{0}$ as a function of $r$. In our configuration, for the case corresponding to figure 5, we obtained for the marginal absolute instability $r_{C A}=22.5, \omega_{0, r}\left(r_{C A}\right)=2.5 \times 2 \pi$ and $k_{0, i}\left(r_{C A}\right)=3.35$, to be compared with Lingwood's $r_{C A}=22.4, \omega_{0, r}\left(r_{C A}\right)=2.82 \times 2 \pi$ and $k_{0, i}\left(r_{C A}\right)=3.41$ for the single disk. Consequently, local stability properties of our flow appear to be the same as those of the classical single-disk flow, so Lingwood's results for local stability will be used hereafter. Note that at very high Reynolds number this similarity would not hold due to 

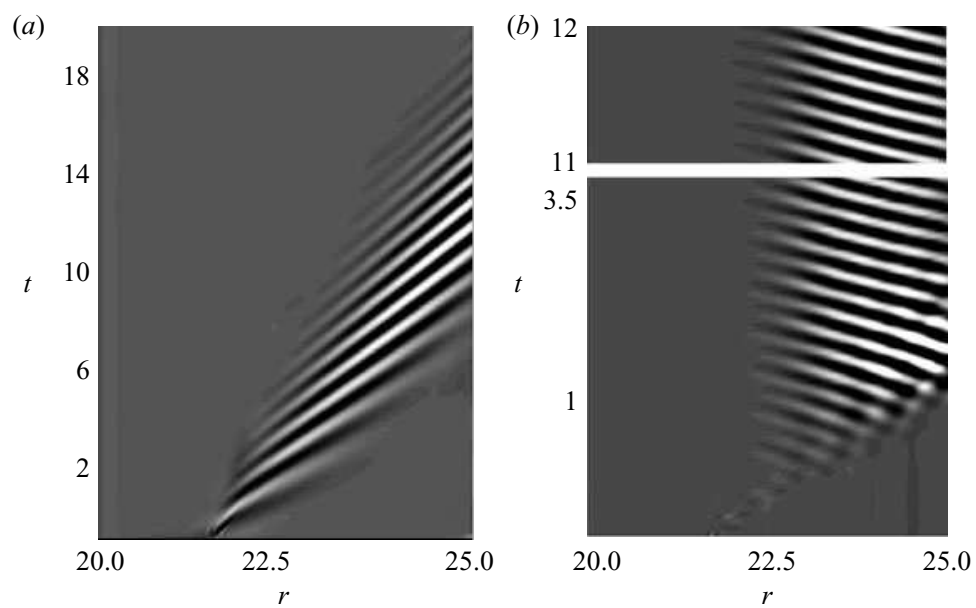

FIGURE 4. Impulse response: Spatio-temporal evolution of the axial velocity for given azimuth $\theta=0$ and height $z=0.42$ in the rotating-disk boundary layer. ( $a)$ Globally stable case $\left(C_{w}=500\right.$, corresponding to $\left.R e_{\delta} \in[94,118], R o \in[-0.22,-0.14]\right)$. (b) Globally unstable case $\left(C_{w}=2500\right.$, $\left.\operatorname{Re}_{\delta} \in[390,490], R o \in[-0.91,-0.64]\right)$.

the confinement effects described in Healey (2007), which might enhance absolute instability.

\subsection{Global nonlinear three-dimensional dynamics}

The three-dimensional dynamics of the fully non-parallel flow has been analysed by superimposing an initial localized finite-amplitude disturbance on the steady axisymmetric base flow, mimicking the sudden intrusion of a roughness at the wall. A Stokes flow over hemispherical obstacles located at the upper wall was used as an initial perturbation field in order to keep the velocity field divergence free. To excite non-zero azimuthal wavenumbers, the obstacle was repeated periodically in the azimuth at the same radial position. In that case, due to the non-zero mean value over the azimuthal direction of the perturbation velocity, axisymmetric modes of instability were also excited as a by-product. However, they were observed always to relax to zero during the computations, in agreement with their convective nature even when the perturbation was nonlinear. The intensity of the perturbation was parametrized by the radius $\left(R_{p}\right)$ of the obstacle, ranging from $R_{p} / h=0.00005$ to $R_{p} / h=0.05$. In most cases we used $R_{p} / h=0.008$. The influence of the magnitude of $R_{p}$ will be discussed later, when investigating the linear global stability of the flow.

Measurements of spatio-temporal characteristics of the travelling spiral vortices were conducted by recording the axial velocity field at each time step, for every radius but at a single azimuth $\theta=0$ and a single height $z=0.42$, corresponding to the position of maximum perturbation velocity. Examples of such data sets are represented in figures $4(a)$ and $4(b)$. The results for radial wavelength $\lambda / \delta$, frequency $\omega_{r}$ and wave angle $\epsilon$ are summarized in table 1, which compares them with a compilation of linear stability analysis (LSA) results from the literature, (see Serre, Tuliszka-Sznitko \& Bontoux 2004).

\subsubsection{Globally stable flow}

The first simulations were carried for small throughflow $C_{w}$ associated with ranges of local Rossby and Reynolds numbers such that, following the linear stability 


\begin{tabular}{lccc}
\multicolumn{1}{c}{ Type } & $\lambda / \delta$ & $\epsilon^{o}$ & $\omega_{r}$ \\
I convective (present results) & 13 & +7 & 1.9 \\
I convective (LSA) & {$[11.45 ; 15.3]$} & {$[7.2 ; 10.9]$} & {$[2.17 ; 5.85]$} \\
II convective (present results) & 22 & -20 & 9 \\
II convective (LSA) & {$[21.66 ; 28.56]$} & $-23.3 ;-26.3]$ & {$[8.17 ; 10.56]$} \\
I absolute $(\beta=68)$ (present results) & 29 & +30 & $2.75 \times 2 \pi$ \\
I absolute $(\beta=68)$ (LSA $R=-1)$ & 28.95 & +31.9 & $2.82 \times 2 \pi$
\end{tabular}

TABLE 1. Mode characteristic parameters.

analysis of Lingwood, the flow was convectively unstable everywhere in the cavity. In this range of control parameters, the flow exhibited a convectively unstable behaviour and remained globally stable as shown by figure 4(a). All the characteristic parameters of type I and type II convective instabilities have been measured (table 1) and found to match those of previous studies; see Serre et al. (2004). Moreover, critical Reynolds numbers corresponding to each type and mode were evaluated by doing successive computations with increasing values of $C_{w}$ and assessing the presence of each type and mode of instability, and they were found to agree with Lingwood (1997).

\subsubsection{Globally unstable flow}

For larger throughflow for which the local Reynolds number is above the absolute threshold in a particular region of the cavity, the upstream edge of the wave packet slows down and stabilizes at a fixed radius while the downstream wave saturates. The theoretical most unstable azimuthal wavenumber according to linear stability analysis is generally agreed to be $n=68$. The first computations were conducted in a full $(2 \pi)$ cavity, and showed that this azimuthal wavenumber is effectively globally unstable in our configuration for the expected values of the control parameters. Further calculations presented here have been performed in a single $2 \pi / 68$ sector. This enables us first to isolate the linear dynamics of a single wavenumber, which when considering the rotational invariance of the flow, interacts only with its harmonics, and second to increase the resolution in the azimuthal direction, thereby increasing the number of harmonics resolved. Indeed, nonlinearities associated with the spiral saturated wave transfer energy to small azimuthal scales that need to be resolved without aliasing.

For $C_{w}=2500$, the flow settled into a nonlinear global mode as demonstrated by figure $4(b)$. The flow structure is characterized by rotating spiral arms shown in figure $5(a)$. The computations have been carried over a time long enough to obtain a steady amplitude profile (figure $5 b$ ) where the amplitude $\sqrt{E(r)}$ is defined as the square root of the kinetic energy contained in the perturbation field at each radial station:

$$
E(r)=r \int_{0}^{2 \pi} \int_{-1}^{1} \frac{1}{2}\left(u^{\prime 2}+v^{\prime 2}+w^{\prime 2}\right) \mathrm{d} z \mathrm{~d} \theta,
$$

$u^{\prime}, v^{\prime}$ and $w^{\prime}$ representing the perturbation velocity with respect to the axisymmetric base flow. The amplitude profile of the spiral mode is remarkable since it is made up of an upstream region where the amplitude is $10^{-4}$ lower than the saturation level downstream, and a middle exponentially growing region followed by a saturated wave. From figure $4(b)$ we measure a dominant frequency of the mode independent of the radial station equal to $\omega_{G} / 2 \pi=2.75$. 
(a)

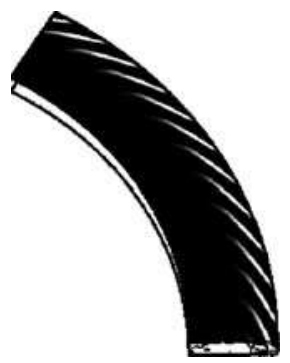

(b)

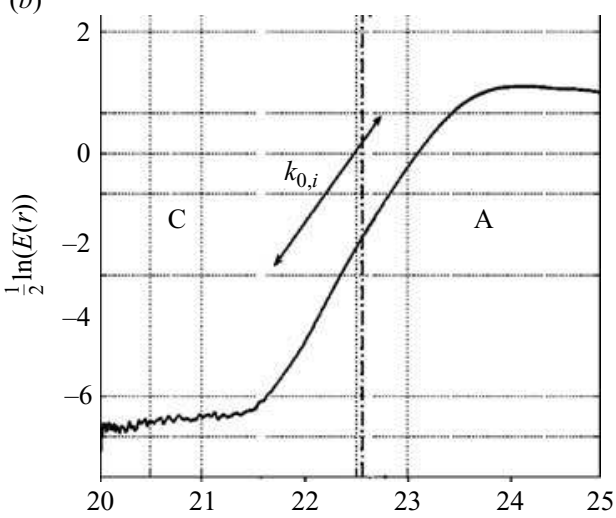

Figure 5. The $n=68$ elephant global mode. ( $a$ ) Three-dimensional view of the spiral vortices corresponding to the final state of figure $4(b) . L=2.5$ and $R_{m}=9$ so that $R_{\text {in }}=20$ and $R_{\text {out }}=25$. (b) Log-linear plot of the local amplitude of the global mode (3.1) as a function of the radial coordinate for $C_{w}=2500$ and $\operatorname{Re}_{h}=780\left(\operatorname{Re}_{\delta} \in[390,490], R o \in[-0.91,-0.64]\right)$. C denotes the convectively unstable region and $\mathrm{A}$ the absolutely unstable region. The location $r_{C A}$ of the convective to absolute transition point for the azimuthal wavenumber $n=68$ determined through interpolation of Lingwood's results is represented by the vertical dash-dotted line. The theoretical absolute spatial growth rate $k_{0, i}$ at the transition point is indicated by its slope.

\subsubsection{Comparisons with elephant mode theory}

The structure described above is the elephant mode structure described by Pier et al. (1998). The dash-dotted vertical line in figure 5(b) represents the upstream boundary of the absolutely unstable domain, estimated by determining at each radial location the local Reynolds and Rossby numbers and interpolating Lingwood's results obtained for the von Kármán profiles over an infinite disk. Even though very few Rossby numbers have been computed in Lingwood (1997), the transition (dashed line on figure $5 b$ ) from convective to absolute instability is centred on the exponentially growing region of the nonlinear global mode. For the present azimuthal wavenumber $n=68$, the interpolated local absolute growth rate $\omega_{0, i}$ becomes positive, i.e. the instability is absolute, after $r_{C A}=22.53$. At this threshold the absolute frequency interpolated from Lingwood's results, $\omega_{0}=2.82 \times 2 \pi$, is in excellent agreement with the nonlinear global mode frequency we measure, $\omega_{G}=2.75 \times 2 \pi$, and the radial absolute wavenumber $k_{0, r}$ corresponds to a spiral pitch of $\epsilon=31^{\circ}$, also very close to the global mode spiral inclination of $\epsilon=30^{\circ}$ (figure $5 a$ ). Last but not least, the absolute spatial growth rate $k_{0, i}=3.41$, indicated by the slope on figure $5(b)$, matches the exponential growth of the global mode $k_{f}=3.63$. The nonlinear global mode observed in the DNS is therefore an elephant mode arising at the upstream boundary of the absolute domain: a front located at $r_{C A}$ plays the role of a wave maker triggering a saturated wave downstream, which beats at the local absolute frequency determined at $r_{C A}$.

\subsection{Global linear response}

In our simulations we have tested low-amplitude initial wave packets by lowering the values of $R_{p}$. The outcome of a simulation carried with such a small perturbation is shown in figure 6 , together with a similar plot for the nonlinearly globally unstable case of figure 5. On these plots the very early peak in amplitude is due to the 


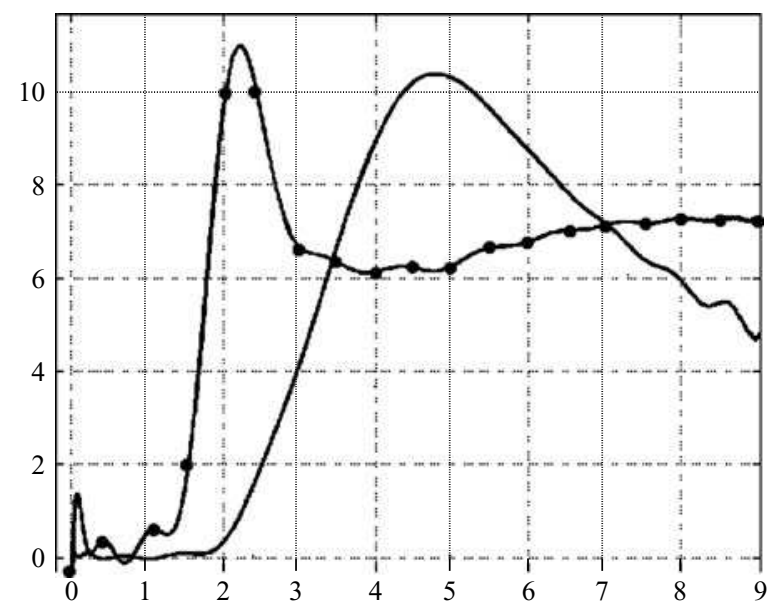

FIgURE 6. Amplitude response in time for the axial perturbation velocity. Configuration and control parameters are those of figure 5. The line with dots corresponds to the nonlinear response shown on figure 5 , and obtained for $R_{p}=0.008$. The plain line is the response obtained with $R_{p}=0.0008$, ten times magnified.

simultaneous excitation of the convective modes. For large-amplitude perturbations, the response levels out at a finite value whereas when the initial perturbation is ten times smaller the response keeps decreasing after the initial transient and vanishes at large time not shown on the figure. The difference in behaviour is sharp, and it indicates that our flow is linearly globally stable but nonlinearly globally unstable.

\section{Conclusion and discussion}

Solutions of the three-dimensional nonlinear Navier-Stokes equations have been investigated in a source-sink flow between two rotating disks. The non-parallel base flow consists of two boundary layers separated by a central core locally in solid-body rotation.

Local stability properties are shown to be the same as those of the single-disk flow for the local values of the Reynolds and Rossby numbers deduced from Lingwood (1997). The corresponding local velocity profile can become absolutely unstable when the local Reynolds number exceeds a threshold value that depends on the local Rossby number. Considering the global dynamics, we show here that, when the computational domain contains a locally absolutely unstable region, a nonlinear selfsustained mode can be observed, and that its structure corresponds to a so-called elephant mode (Pier \& Huerre 2001a) at the boundary of the absolute domain, i.e. a wave evanescent upstream and saturated downstream of this location. In remarkable agreement with the theory of Pier et al. (1998), the global frequency $\left(\omega_{G}=2.75 \times 2 \pi\right)$ and the front steepness $\left(k_{f}=3.63\right)$ are close to the absolute frequency at the transition $\left(\omega_{0, r}=2.8 \times 2 \pi\right)$ and the absolute spatial growth rate $\left(k_{0, i}=3.41\right)$, both estimated from Lingwood (1997) and the present local stability analysis. This global bifurcation was found to be subcritical since the flow remains globally stable for small-enough initial perturbations. In the WKB approximation, this stabilization of an absolutely unstable flow through non-parallel effects is due to $\omega_{0, r}$ variations. This stabilizing effect, depending on the way in which $\omega_{0, r}$ varies with $r$, is not a general feature, but seems to be valid for both the single- and the double-disk configurations. 
In the single-disk case Davies \& Carpenter (2003), Davies et al. (2007) and Othman \& Corke (2006) have shown that the non-parallel effects stabilize the linear global mode, whereas Pier (2003) has proposed that nonlinearities give rise to a nonlinear (elephant) global mode triggered by the absolute transition. Othman \& Corke (2006) used a stronger perturbation too, in order to investigate the nonlinear behaviour. However, this proved less conclusive than the linear case, and they conclude that 'they do not have sufficient evidence to address the question of whether the higher-amplitude condition triggered a global mode.'

For a single disk in an otherwise still fluid, the existence of a nonlinear global mode that would imply a subcritical global bifurcation is still an open issue. Such a subcritical global bifurcation scenario is fully established for the present flow between two disks. The confinement mechanism described by Healey (2007) and Juniper (2006) appears not to act, since local properties are unaffected. However, although we have shown that the local properties of both flows are similar in the Reynolds number range considered here, one should keep in mind that non-parallel effects differ. In the cavity flow, the geostrophic velocity varies like $1 / r$ whereas for the single disk it is proportional to $r$. However, in the WKB approximation elephant modes are not sensitive to non-parallel effects at leading order, the dynamics of such modes being led by the front, acting as a wave maker, which is a very local phenomenon. They are determined only by the characteristics at the upstream boundary of the absolutely unstable domain, namely by $\omega_{0}$ at $r=r_{C A}$. This prediction is fully confirmed in the double-disk case, making its applicability to the single-disk case highly probable. Even if it were so, the transition to the turbulence scenario for the double- and the single-disk cases may differ and genericity of any particular mechanism would have to be discussed with care. In particular, experimental results of Othman \& Corke (2006) showing that a reduction of the flow perturbations delays the transition to turbulence to larger radius, i.e. a larger Reynolds number, question the absolute secondary instability scenario proposed by Pier (2003). Further studies of the present doubledisk geometry may contribute to this debate since the existence of the elephant mode, which is a prerequisite for the absolute secondary instability scenario, is established. The present proof of the existence of an elephant mode in the double-disk case therefore strongly favours of its existence in the single-disk case.

It remains for future works to numerically compute the transition to turbulence in the double-disk case and to analyse the role of the nonlinear global mode in such a transition. Results of Pier (2003) on the local stability properties of spiral waves should also apply to the double-disk case. It should be therefore possible to test the direct route to turbulence that Pier proposed through secondary instability of the nonlinear global mode.

It should be stated that at this stage our simulations cannot be conclusive about the validity of Pier's scenario. In our computations the restriction of the possible azimuthal wavenumbers due to the sectorial cavity prevents subharmonic secondary instability. This could be the reason why we can observe the sustained presence of the global mode. Regarding experiments, if the global mode is effectively already absolutely unstable as soon as it forms, it probably will not be seen.

More intensive computations are required to investigate a possible secondary global instability, in the form of a second-generation elephant mode riding on the back of the first one, and leading to disorder.

All computations were carried on the NEC SX-8 from IDRIS (project 060242). Constructive interactions with the referees are warmly acknowledged. 


\section{REFERENCES}

Andersen, A., Lautrup, B. \& Bohr, T. 2003 Averaging method for the nonlinear laminar Ekman layers. J. Fluid Mech. 487, 81-90.

Bassom, A. P., Kuzanyan, K. M. \& Soward, A. M. 1999 A nonlinear dynamo wave riding on a spatially varying background. Proc. R. Soc. Lond. A 455, 1443-1481.

Brancher, P. \& Chomaz, J. M. 1997 Absolute and convective secondary instabilities in spatially periodic shear flows. Phys. Rev. Lett. 78, 658-661.

Chomaz, J. M. \& Couarron, A. 1999 Against the wind. Phys. Fluids 11, 2977-2983.

Chomaz, J. M., Couairon, A. \& Julien, S. 1999 Absolute and convective nature of the Eckhaus and zigzag instability with throughflow. Phys. Fluids 11, 3369-3373.

Couairon, A. \& Chomaz, J. M. 1997 Pattern selection in the presence of a crossflow. Phys. Rev. Lett. 79, 2666-2669.

Couarron, A. \& Chomaz, J. M. 1999a Fully nonlinear global modes in slowly varying flows. Phys. Fluids 12, 3688-3703.

Couairon, A. \& Chomaz, J. M. $1999 b$ Primary and secondary nonlinear global instability. Physica D 132, 428-456.

Couairon, A. \& Chomaz, J. M. 2001 Pushed global modes in weakly inhomogeneous subcritical flows. Physica D 158, 129-150.

Crespo del Arco, E., Maubert, P., Randriamampianina, A. \& Bontoux, P. 1996 Spatio-temporal behaviour in a rotating annulus with a source-sink flow. J. Fluid Mech. 32, 1-27.

Crespo del Arco, E., Serre, E., Bontoux, P. \& Launder, B. E. 2005 Stability, transition and turbulence in rotating cavities. In Advances in Fluid Mechanics (ed. M. Rahman), vol. 41. WIT press.

Davies, C. \& CARPenter, P. W. 2003 Global behaviour corresponding to the absolute instability of the rotating-disk boundary layer. J. Fluid Mech. 486, 287-329.

Davies, C., Thomas, C. \& Carpenter, P. W. 2007 Global stability of the rotating-disk boundary layer. J. Engng Maths 57, 219-236.

Delbende, Y., Chomaz, J. M. \& Huerre, P. 1998 Absolute/convective instabilities in the batchelor vortex: a numerical study of the linear impulse response. J. Fluid Mech. 355, 229-254.

Healey, J. J. 2007 Enhancing the absolute instability of a boundary layer by adding a far-away plate. J. Fluid Mech. 579, 29-61.

Hide, R. 1968 On source-sink flows stratified in a rotating annulus. J. Fluid Mech. 32, 737-764.

HuERre, P. 1988 On the absolute/convective nature of primary and secondary instabilities. In Propagation in Systems Far From Equilibrium (ed. J. E. Wesfreid, H. R. Brand, P. Manneville, G. Albinet \& N. Boccara). Springer.

JUNIPER, M. P. 2006 The effects of confinement on the stability of two-dimensional shear flows. J. Fluid Mech. 565, 171-195.

von Kármán, Th. 1921 Über laminare und turbulente Reibung. Z. Angew. Math. Mech. 1, 233-252.

KocH, W. 2002 On the spatio-temporal stability of primary and secondary crossflow vortices in a three-dimensional boundary layer. J. Fluid Mech. 456, 85-111.

Lingwood, R. J. 1995 Absolute instability of the boundary layer on a rotating disk. J. Fluid Mech. 299, 17-33.

LingwOOD, R. J. 1996 An experimental study of the absolute instability of the rotating-disk boundary-layer flow. J. Fluid Mech. 314, 373-405.

LingwoOD, R. J. 1997 Absolute instability of the Ekman layer and related rotating flows. J. Fluid Mech. 331, 405-428.

MACK, L. M. 1985 The wave pattern produced by a point source on a rotating-disk flow. AIAA Paper 85-0490.

Meunier, N., Proctor, M. R. E., Sokoloff, D. D., Soward, A. M. \& Tobias, S. M. 1997 Asymptotic properties of a nonlinear alpha omega-dynamo wave: Period, amplitude and latitude dependence. Geophys. Astrophys. Fluid Dyn. 86, 249-285.

OthMAN, H. \& CORKE, T. C. 2006 Experimental investigation of absolute instability of a rotating-disk boundary layer. J. Fluid Mech. 565, 63-94.

Owen, J. M. \& Rogers, R. H. 1995 In Heat Transfer in Rotating-Disk System (ed. W. D. Morris), vol. 2. Wiley. 
PIER, B. 2003 Finite amplitude crossflow vortices, secondary instability and transition in the rotatingdisk boundary layer. J. Fluid Mech. 487, 315-343.

Pier, B. \& Huerre, P. $2001 a$ Nonlinear self-sustained structures and fronts in spatially developping wake flows. J. Fluid Mech. 435, 145-174.

Pier, B. \& Huerre, P. $2001 b$ Nonlinear synchronization in open flows. J. Fluids Struct. 15, 471-480.

Pier, B., Huerre, P., Chomaz, J. M. \& Couairon, A. 1998 Steep nonlinear global modes in spatially developping media. Phys. Fluids 10, 2433-2435.

Raspo, I., Hugues, S., Serre, E., Randriamampianina, A. \& Bontoux, P. 2002 A spectral projection method for the simulation of complex three-dimensional rotating flows. Comput. Fluids 31, 745-767.

Ruith, M. R., Chen, E. \& Meiburg, E. 2004 Developement of boundary conditions for dns of three-dimensional vortex breakdown phenomena in semi-infinite domains. Comput. Fluids 33, $1225-1250$.

VAn SaARloos, W. 2003 Front propagation into unstable states. Phys. Rep. Rev. 386, 29-222.

Serre, E., Hugues, S., Crespo del Arco, E., Randriamampianina, A. \& Bontoux, P. 2001 Axisymmetric and three-dimensional instabilities in an Ekman boundary-layer flow. Intl J. Heat Fluid Flow 22, 82-93.

Serre, E., Tuliszka-Szzitko, E. \& Bontoux, P. 2004 Coupled numerical and theoretical study of the flow transition between a rotating and a stationary disk. Phys. Fluids 16, 688-706.

Tobias, S. M., Proctor, M. R. E. \& Knobloch, E. 1997 The role of absolute instability in the solar dynamo. Astron. Astrophys. 318, L55-L58.

Tobias, S. M., Proctor, M. R. E. \& Knobloch, E. 1998 Convective and absolute instabilities of fluid flows in finite geometry. Physica D 113, 43-72.

Turkyilmazoglu, M. \& GaJJaR, J. S. B. 2000 Direct spatial resonnance in the laminar boundary layer due to a rotating disk. Sadhana-Acad. Proc. 25, 601-617. 